\title{
Two-stage association tests for genome-wide association studies based on family data with arbitrary family structure
}

\author{
Tao Feng ${ }^{1,2}$, Shuanglin Zhang ${ }^{1,2}$ and Qiuying Sha ${ }^{*, 1}$ \\ ${ }^{1}$ Department of Mathematical Sciences, Michigan Technological University, Houghton, MI, USA; ${ }^{2}$ Department of \\ Mathematics, Heilongjiang University, Harbin, China
}

Recently, Steen et al proposed a two-stage approach for genome-wide family-based association studies. In the first stage, a screening test is used to select markers, and in the second stage, a family-based association test is performed on a much smaller set of the selected markers. The two-stage method can be much more powerful than the traditional family-based association tests. In this article, we extend the approach so that it can incorporate parental information and can be applied to an arbitrary pedigree structure. We use simulation studies to evaluate the type I error rates and the power of the proposed methods. Our results show that the two-stage approach that incorporates founders' phenotypes has the correct type I error rates, and is much more powerful than the two-stage approach that uses children's phenotypes only. Also, by carefully choosing the number of markers retained in the first stage, the power of a two-stage approach can be much more than that of the corresponding one-stage approach. European Journal of Human Genetics (2007) 15, 1169-1175; doi:10.1038/sj.ejhg.5201902; published online 25 July 2007

Keywords: two-stage design; genome-wide association study; family-based association test

\section{Introduction}

Rapid progress in high-throughput genotyping technologies has greatly reduced the cost of genome-wide analyses and is resulting in a boom of large-scale genetic association studies on common disorders. Genome-wide association studies are now widely used approaches in the search for loci that affect complex traits. While genome-wide association studies are promising to mapping complex disease genes, ${ }^{1-3}$ their success will depend on whether the information gain of the increased number of SNPs will be diluted by the multiple-comparison problem. ${ }^{1}$ When hundreds of thousands of SNPs are tested for association,

${ }^{*}$ Correspondence: Dr Q Sha, Department of Mathematical Sciences, Michigan Technological University, 1400 Townsend Drive, Houghton, MI 49931, USA.

Tel: + 1906487 2146; Fax: + 1906487 3133;

E-mail: qsha@mtu.edu

Received 11 April 2007; revised 13 June 2007; accepted 14 June 2007; published online 25 July 2007 the $P$-values need to be adjusted for controlling type I error rates. Most multiple-testing adjustment approaches, including the Bonferroni correction for controlling familywise error rate and the method proposed by Benjamini and Hochberg ${ }^{4}$ for controlling false discovery rate (FDR), become more conservative as more tests are performed.

In case-control studies, several authors have proposed a two-stage design that utilizes two samples. 5 The first sample is used to screen and select SNPs for association tests. The association tests are conducted on the selected SNPs by using the second sample, so that the number of association tests is diminished and the correction for multiple testing is less severe. To avoid biased test results, the sample used in the screening step should be independent of the sample used for association testing. For family data, it is possible to create two sources of information using one sample. ${ }^{7}$ Recently, in mapping quantitative trait loci using family data, Steen et al ${ }^{1}$ proposed an interesting approach that performs the SNP screening and association 
test using the same sample. The basic idea of Steen et al's method is that the screening test based on the traits of the children and between-family genotype scores is statistically independent of the association test that depends on children's trait values and within-family genotype scores. The screening test is used first to select SNPs, and a familybased association test is performed on a much smaller set of the selected SNPs. Since the significance of the association is decided by a transmission/disequilibrium test (TDT) type family-based association test, the results are robust to population admixture.

In this article, motivated by Steen et al's method, we propose two two-stage approaches to analyze genome-wide association data from general pedigrees. The two approaches use two different screening tests in the first stage, but they use the same quantitative pedigree disequilibrium test (QPDT) proposed by Zhang et $\mathrm{al}^{8}$ as the association test in the second stage. The first screening test, similar to that used by Steen et al, ${ }^{1}$ does not use parental phenotypes. The second screening test uses phenotypes of the founders in the pedigrees. We use simulation studies to evaluate the performance of the two approaches. Our simulation studies show that by using part of the parental phenotypes, the second approach is consistently more powerful than the first approach, which does not use parental information. Furthermore, by carefully choosing the number of selected SNPs in the first stage, both approaches are more powerful than the one-stage approach that does not use screening tests.

\section{Methods}

For the two-stage approaches, if the association test used in the second stage is robust to population admixture, then the two-stage approach will be robust to population admixture regardless of the screening test used in the first stage. Because the number of markers returned in the first step, denoted by $L$, is fixed in advance and the critical value at the stage is set accordingly, even if the set of $L$ markers tested in the second stage includes a high proportion of false positives due to population stratification, the proportion of those that pass the second stage is not inflated by population stratification and the overall probability that a marker would pass both stages is the nominal test size. In this section, we will propose two twostage approaches for genome-wide family-based association studies. We will first describe the QPDT, the association test used in the second stage for both the two proposed two-stage approaches.

QPDT: the association test used in the second stage The $\mathrm{TDT}^{9}$ and its extensions for qualitative traits ${ }^{10-14}$ and quantitative traits ${ }^{15-19}$ have become popular tools in testing linkage and association. The main advantage of these TDT type methods is the robustness to population admixture. For quantitative traits, we chose the QPDT as the association test in our proposed two-stage approaches because it allows arbitrary pedigree structures. The QPDT, proposed by Zhang et al, ${ }^{8}$ divides each pedigree into several nuclear families and combines the information from the nuclear families. The QPDT mainly uses the following two types of informative nuclear families in an extended pedigree:

i Families with both parents available and with at least one parent being heterozygous at the marker being studied.

ii Families with at most one available parent and with multiple offspring where at least two siblings have different genotypes.

Assume that there are $n$ pedigrees, $n_{i}$ informative nuclear families in the ith pedigree, and $n_{i j}$ children in the $j$ th informative nuclear family of the $i$ th pedigree. Consider a biallelic marker with two alleles 0 and 1 . We use the following notation:

- $Y_{i j \mathrm{~F}}, Y_{i j \mathrm{M}}$, and $Y_{i j k}$ : the trait value of the father, mother, and the $k$ th child in the $j$ th informative nuclear family of the $i$ th pedigree, respectively.

- $X_{i j \mathrm{~F}}, X_{i j \mathrm{M}}$, and $X_{i j k}$ : the genotypic score (number of copies of alleles 1) of the father, mother, and the $k$ th child in the $j$ th informative nuclear family of the ith pedigree, respectively.

- $\bar{Y}$ and $\bar{X}$ : The average trait value and average genotypic score of all the individuals, respectively.

- $\bar{X}_{i j}$ : If parental genotypes are available (the first type of informative nuclear families), $\bar{X}_{i j}=\left(X_{i j F}+X_{i j M}\right) / 2$; otherwise (the second type of informative nuclear families),

$$
\bar{X}_{i j}=\frac{1}{n_{i j}} \sum_{k=1}^{n_{i j}} X_{i j k}
$$

By using the notation, the statistic of the QPDT is given by

$$
\mathrm{QPDT}=\frac{\sum_{\mathrm{i}=1}^{\mathrm{n}} \mathrm{U}_{\mathrm{i}}}{\sqrt{\sum_{\mathrm{i}=1}^{\mathrm{n}} \mathrm{U}_{\mathrm{i}}^{2}}},
$$

where

$$
U_{i}=\sum_{j=1}^{n_{i}} U_{i j}
$$

and $U_{i j}=\frac{1}{n_{i j}} \sum_{k=1}^{n_{i j}}\left(Y_{i j k}-\bar{Y}\right)\left(X_{i j k}-\bar{X}_{i j}\right)$. Under the null hypothesis of no linkage disequilibrium, the QPDT asymptotically follows a standard normal distribution. We can think of random variables $U_{i j}$ as the covariance between the children's trait values and within-family genotypic score, $X_{i j k}-\bar{X}_{i j}$. The QPDT only uses the within-family genotype information, and thus is robust to population admixture. 
The screening tests used in the first stage

Although the QPDT only uses informative nuclear families, the screening tests can use all the nuclear families in a pedigree. Let $N_{i}$ denote the number of nuclear families in the $i$ th pedigree. The two screening test statistics are in the same form as the QPDT statistic. Both the two screening test statistics can be written as

$$
T_{\text {SCREEN }}=\frac{\sum_{i=1}^{n} U_{i}}{\sqrt{\sum_{i=1}^{n} U_{i}^{2}}},
$$

where

$$
U_{i}=\sum_{j=1}^{N_{i}} U_{i j}
$$

However, $U_{i j}$ are different in the two tests. The definitions of $U_{i j}$ in the two tests are given below:

1. In the first screening test, which uses children's phenotypes only,

$$
U_{i j}=\frac{1}{n_{i j}} \sum_{k=1}^{n_{i j}}\left(Y_{i j k}-\bar{Y}\right)\left(\bar{X}_{i j}-\bar{X}\right)
$$

In this case, the corresponding two-stage approach is called a two-stage test using children's phenotypes only (TTCPO).

2. In the second screening test, which uses children's phenotypes as well as the phenotypes of founders of the pedigrees,

$$
\begin{aligned}
U_{i j}= & \frac{1}{n_{i j}} \sum_{k=1}^{n_{i j}}\left(Y_{i j k}-\bar{Y}\right)\left(\bar{X}_{i j}-\bar{X}\right) \\
& +\left(Y_{i j \mathrm{~F}}-\bar{Y}\right)\left(X_{i j \mathrm{~F}}-\bar{X}\right) I_{i j \mathrm{~F}} \\
& +\left(Y_{i j \mathrm{M}}-\bar{Y}\right)\left(X_{i j \mathrm{M}}-\bar{X}\right) I_{i j \mathrm{M}}
\end{aligned}
$$

where $I_{i j \mathrm{~F}}=1$ if the father of the $j$ th family in the $i$ th pedigree is a founder of this pedigree; otherwise, $I_{i j \mathrm{~F}}=0$ and $I_{i j \mathrm{M}}$ is similarly defined for the mother. In this case, the corresponding two-stage approach is called a twostage test using founder's phenotypes (TTFP).

Under the null hypothesis of no association, both the screening test statistics follow a standard normal distribution.

\section{Applying the two-stage approaches to genome-wide} association studies

Suppose that $M$ SNPs have been genotyped for each sampled individual. In the first stage, we use one of the screening tests to test each of the $M$ SNPs. Select $L$ SNPs with the smallest p-values, where $L$ is a pre-specified number (we will discuss how to choose $L$ later). In the second stage, we apply the QPDT to the $L$ selected SNPs, and declare an SNP as significant at a level of $\alpha$ if the pvalue of the QPDT at this marker is less than a threshold $\delta_{L \alpha}$. The threshold $\delta_{L \alpha}$ is determined by controlling the FDR, the ratio of the number of falsely rejected null hypotheses to the total number of rejected null hypo- theses, at a level of $\alpha$. To control the FDR at a level of $\alpha$, we can choose the cutoff $\delta_{L \alpha}$ as follows: ${ }^{4}$

let $p_{(1)}, \ldots, p_{(\mathrm{L})}$ be the ordered $p$-values when we apply the PDT to the $L$ selected markers; then $\delta_{L \alpha}=\max \left\{p_{(\mathrm{i})}: p_{(\mathrm{i})} \leqslant \mathrm{i} \alpha / L\right\}$.

\section{Results}

We use simulation studies to evaluate the performance of the proposed methods. In the simulations, we consider two family structures. The first one is a nuclear family with one child; the second one is a three generation pedigree as given in Figure 1. This family structure is one of the Centre $\mathrm{d}^{\prime}$ Etude du Polymorphism Humans (CEPH) pedigrees. ${ }^{20}$ For each family structure, we use two approaches to generate genotype data. In the first approach, we generate the genotypes of the founders by assuming Hardy-Weinberg equilibrium and linkage equilibrium. Each founder randomly transmits one of his/her two alleles to form the children's genotypes. This means that we generate each allele and each marker independently. The frequency of the minor allele at each marker is a random number between 0.05 and 0.45 .

In the second approach, we want to mimic the linkage disequilibrium (LD) structure of real data sets. To generate the genotypes with LD between markers, we use Hudson's ms program, ${ }^{21}$ which assumes the coalescent process with recombination to generate multi-marker haplotypes. We follow Nordborg M, Tavare S, ${ }^{22}$ and Kimmel G and Shamir $\mathrm{R},{ }^{23}$ using a mutation rate of $2.5 \times 10^{-8}$ per nucleotide per generation, a recombination rate of $10^{-8}$ per pair of nucleotides per generation, and an effective population size of 10000 . Of all the segregating sites, only the ones with a minor allele frequency $>5 \%$ are defined as SNPs and are used in the rest of the analysis. The simulation program produces populations of haplotypes from which genotypes of the founders in each pedigree can be generated. The genotypes for each individual who is not a founder can be formed by her/his parents.

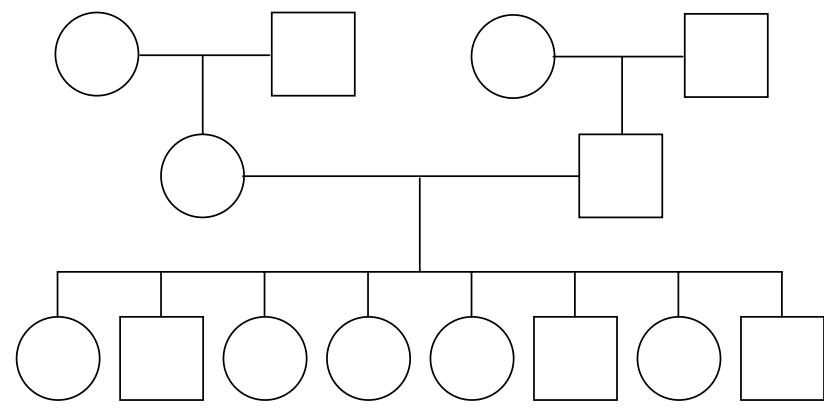

Figure 1 The pedigree structure used in the simulation studies. 


\section{Type I error rates}

To assess the type I error rates, we generate data under the null hypothesis of no association. Let $Y_{1}=\left(y_{\mathrm{F}}, y_{\mathrm{M}}\right)$ denote the trait values of the parents and $Y_{2}=\left(y_{1}, y_{2}, \ldots, y_{m}\right)$ denote the trait values of the $m$ children. Assume that $\left(Y_{1}, Y_{2}\right)$ follows a normal distribution with a mean vector of zero and variance-covariance matrix of

$$
\begin{aligned}
& \sum=\left(\begin{array}{ll}
\sum_{11} & \sum_{12} \\
\sum_{21} & \sum_{22}
\end{array}\right) \text { where } \sum_{11}=\left(\begin{array}{cc}
1 & 0 \\
0 & 1
\end{array}\right) \\
& \sum_{12}=\sum_{21}=\left(\begin{array}{lll}
\rho & \ldots & \rho \\
\rho & \ldots & \rho
\end{array}\right) \text { and } \sum_{22}=\left(\begin{array}{lll}
1 & \ldots & \rho \\
& & \\
\rho & \ldots & 1
\end{array}\right)
\end{aligned}
$$

This simulation setting means that the father and mother are independent, and parents with children, and children with children are correlated to the correlation coefficient of $\rho$. The conditional distribution of $Y_{2}=\left(y_{1}, \ldots, y_{m}\right)$ given parental trait values $Y_{1}=\left(y_{F}, y_{M}\right)$ is a normal distribution with mean vector of

$$
\mu_{c}=\sum_{21} \sum_{11}^{-1} Y_{1}
$$

and variance-covariance matrix of

$$
\sum_{c}=\sum_{22}-\sum_{21} \sum_{11}^{-1} \sum_{12} .
$$

To generate trait values of all individuals in a pedigree, we first generate the trait value of each founder by using a standard normal distribution. The trait values of the other members can be generated by a normal distribution with a mean vector of $\mu_{c}$ and variance-covariance matrix of $\sum_{c}$, given the trait values of their parents.

We consider sample sizes of 200,400,600, and 800 for nuclear families, and sample sizes of 50, 100, 200, and 300 for large pedigrees. We also consider three different numbers of markers: 1000, 10000, and 100000 . For each simulation scenario, we generate 1000 samples to estimate type I error rates of the two two-stage approaches by using different values of $L$ (the number of markers retained in the first stage). Note that when $L=M$ (the total number of markers), the two-stage approach is equivalent to the onestage approach. For 1000 replications, the 95\% confidence interval of type I error rate is $(0.0362,0.0638)$ for the nominal level of 5\%. The type I error results based on genotypes generated under Hardy-Weinberg equilibrium are similar to those based on genotypes generated by ms program. ${ }^{21}$ We only report the results based on genotypes generated by ms program. The estimated type I error rates are summarized in Table 1 for nuclear families and Table 2 for large pedigrees. The two tables show that the type I error rates of the TTCPO and TTFP are very consistent with the nominal level of $5 \%$ for a small value of $L$ and little more conservative for a larger $L$. The conservativeness of type I error rates for a large value of $L$ is because of the conservative nature of multiple test adjustments by controlling the FDR. The type I error rates for the TTCPO
Table 1 Type I error rates (in percentage) of the two twostage tests based on nuclear family samples

\begin{tabular}{lrcccccc}
\hline & & \multicolumn{3}{c}{ TTCPO } & \multicolumn{4}{c}{ TTFP } \\
& & \multicolumn{3}{c}{$L$} & & & $L$ \\
Sample size & Number of markers & 1 & 10 & 100 & 1 & 10 & 100 \\
\hline 200 & 1000 & 4.8 & 5.2 & 2.8 & 5.3 & 4.6 & 3.3 \\
& 10000 & 4.6 & 4.3 & 2.9 & 5.4 & 4.2 & 2.9 \\
& 100000 & 3.8 & 3.0 & 2.6 & 4.3 & 3.8 & 3.0 \\
400 & 1000 & 5.5 & 5.1 & 3.5 & 5.0 & 4.6 & 3.7 \\
& 10000 & 3.9 & 3.7 & 3.2 & 5.3 & 4.1 & 3.8 \\
600 & 100000 & 6.2 & 5.7 & 4.0 & 5.3 & 4.5 & 4.0 \\
& 1000 & 5.6 & 4.9 & 4.4 & 4.0 & 3.8 & 3.9 \\
& 10000 & 5.3 & 4.0 & 3.8 & 5.3 & 4.7 & 3.4 \\
800 & 100000 & 4.4 & 4.2 & 3.1 & 5.0 & 4.5 & 3.2 \\
& 1000 & 4.7 & 4.8 & 4.6 & 4.2 & 5.7 & 4.6 \\
& 10000 & 5.7 & 3.5 & 3.8 & 4.9 & 4.0 & 5.1 \\
& 100000 & 4.0 & 3.9 & 4.0 & 5.8 & 5.5 & 3.2 \\
\hline
\end{tabular}

This set of simulations uses the genotypes generated by ms software. $L$ denotes the number of markers retained in the first stage.

Table 2 Type I error rates (in percentage) of the two twostage tests based on general pedigree samples

\begin{tabular}{lrrrrrccc}
\hline & & \multicolumn{3}{c}{ TTCPO } & \multicolumn{4}{c}{ TTFP } \\
& & \multicolumn{3}{c}{$L$} & & & $L$ \\
Sample size & Number of markers & 1 & 10 & 100 & 1 & 10 & 100 \\
\hline 200 & 1000 & 4.8 & 5.2 & 2.8 & 5.3 & 4.6 & 3.3 \\
& 10000 & 4.6 & 4.3 & 2.9 & 5.4 & 4.2 & 2.9 \\
& 100000 & 3.8 & 3.0 & 2.6 & 4.3 & 3.8 & 3.0 \\
400 & 1000 & 5.5 & 5.1 & 3.5 & 5.0 & 4.6 & 3.7 \\
& 10000 & 3.9 & 3.7 & 3.2 & 5.3 & 4.1 & 3.8 \\
600 & 100000 & 6.2 & 5.7 & 4.0 & 5.3 & 4.5 & 4.0 \\
& 1000 & 5.6 & 4.9 & 4.4 & 4.0 & 3.8 & 3.9 \\
& 10000 & 5.3 & 4.0 & 3.8 & 5.3 & 4.7 & 3.4 \\
800 & 100000 & 4.4 & 4.2 & 3.1 & 5.0 & 4.5 & 3.2 \\
& 1000 & 4.7 & 4.8 & 4.6 & 4.2 & 5.7 & 4.6 \\
& 10000 & 5.7 & 3.5 & 3.8 & 4.9 & 4.0 & 5.1 \\
& 100000 & 4.0 & 3.9 & 4.0 & 5.8 & 5.5 & 3.2 \\
\hline
\end{tabular}

This set of simulations uses the genotypes generated by ms software. $L$ denotes the number of markers retained in the first stage.

and TTFP also show that the screening tests used in the first stage and the PDT used in the second stage are independent.

\section{Power comparisons}

For power comparisons, we first generate genotype data as described in the previous section and then generate trait values according to different disease models as described below. For a pedigree with $K$ members, let $\varepsilon_{1}, \ldots, \varepsilon_{K}$ be the trait values of the $K$ members generated under the null hypothesis using the method described in the previous section. Assume that the disease locus is a biallelic marker with two alleles $D$ and $d$. Then, the trait value of the $k$ th member is given by

$$
y_{k}=\beta_{1} x_{k 1}+\beta_{2} x_{k 2}+\varepsilon_{k},
$$

where $x_{k 1}$ is the additive genotypic score, that is, $x_{k 1}=-1$, 0 , or 1 corresponding to genotypes $d d, D d$, or $D D$ of the $k$ th 
member; $x_{k 2}$ is the dominant genotypic score, that is, $x_{k 2}=0,1$, or 0 corresponding to genotypes $d d$, $D d$, or $D D$ of the $k$ th member. We consider three disease models: the additive model in which $\beta_{2}=0$, the dominant model in which $\beta_{1}=\beta_{2}$, and the recessive model in which $\beta_{1}=-\beta_{2}$. The values of $\beta_{1}$ and $\beta_{2}$ can be determined by the value of the heritability $h$ and disease models. Consider the additive model as an example. Under the additive model,

$$
\begin{aligned}
& h=\frac{\operatorname{var}\left(\beta_{1} x_{1}\right)}{\operatorname{var}(y)}=\frac{\beta_{1}^{2} \operatorname{var}\left(x_{1}\right)}{\beta_{1}^{2} \operatorname{var}\left(x_{1}\right)+1}=\frac{2 \beta_{1}^{2} p(1-p)}{2 \beta_{1}^{2} p(1-p)+1} \\
& \quad \text { and thus } \\
& \beta_{1}=\sqrt{\frac{h}{2(1-h) p(1-p)}},
\end{aligned}
$$

where $p$ denotes the allele frequency of allele $\mathrm{D}$ at the disease locus.
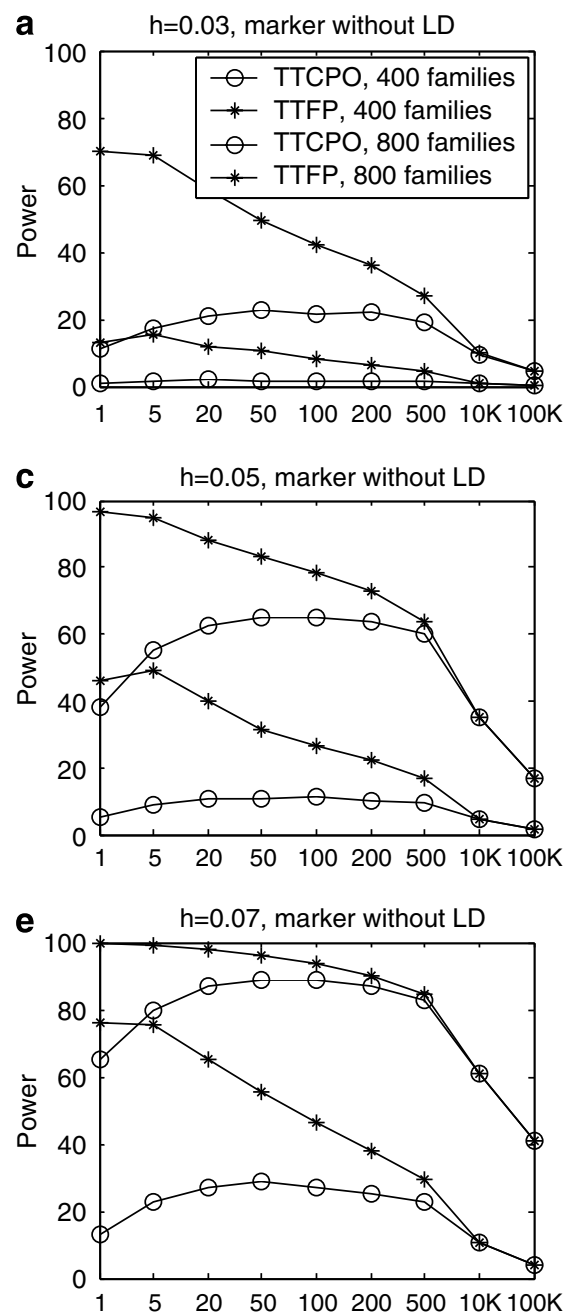

Figure 2 Power comparisons of the two-stage tests using 400 and 800 nuclear families with one child in each family. The $x$-axis denotes the number of markers retained by the screening test in the first stage. The total number of markers is 100000 (100K). Figures in the left panel, $\mathbf{a}, \mathbf{c}$ and $\mathbf{e}$, show powers based on genotype data generated under the assumption of linkage equilibrium between markers. Figures in the right panel, $\mathbf{b}$, $\mathbf{d}$ and $\mathbf{f}$, show powers based on genotype data generated by using ms software. $h$ denotes the heritability.
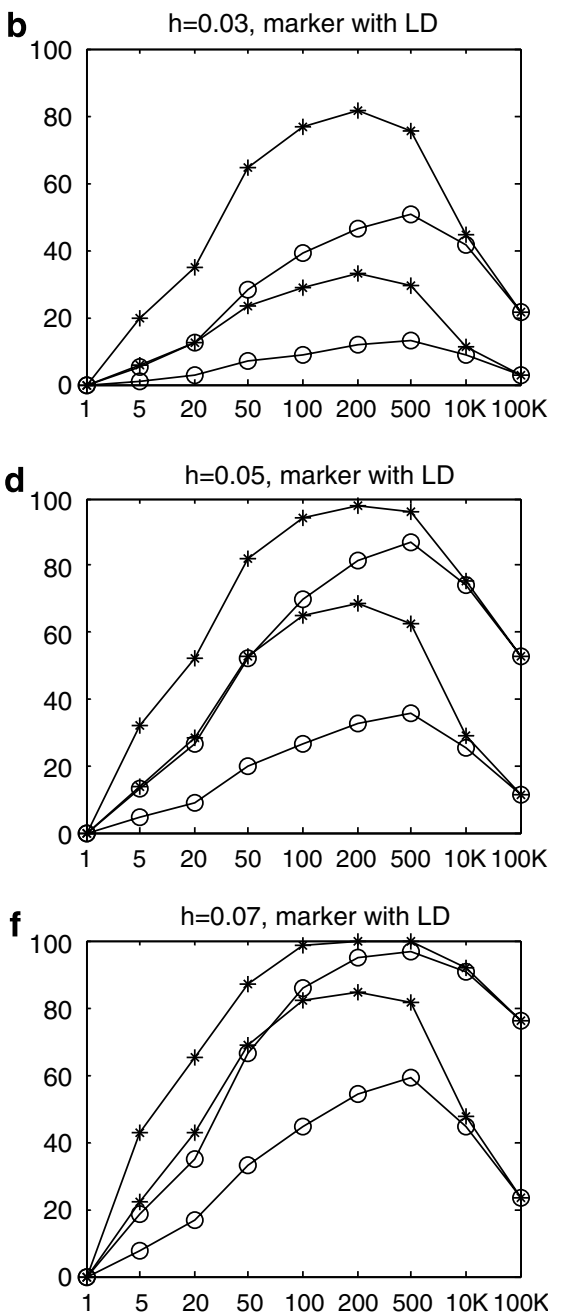

In our simulations, we generate genotypes at 100000 markers for each individual by either one of the two of markers retained in the first step) from 1 to 100000 . The results of $L=100000$ are the results of the one-stage approach, the PDT. We consider different sample sizes, different disease models, and different values of heritability $h$. For each scenario, we use 1000 replicated samples to estimate the power of the tests. To reduce the effect of allele frequency at the disease locus, we randomly choose a marker with minor allele frequency between 0.1 and 0.4 as a disease locus for each replication.

The patterns of power comparisons are similar under the three disease models. We use the additive model as an example to describe the results. The power comparisons of the TTPCO and TTFP are given in Figure 2 for nuclear families and in Figure 3 for large pedigrees. The two figures generating methods, and vary the value of $L$ (the number 
show that the TTFP, which uses the founders' phenotypes consistently outperforms the TTCPO for all the scenarios we considered. In most cases, the power of the TTFP is substantially more than that of the TTCPO. For example, the power of the TTFP is $70 \%$ while the power of the TTCPO is $10 \%$ when the sample contains 800 nuclear families and the heritability $h=0.03$, the number of markers retained in the first stage $L=1$, and the genotypes are generated under the assumptions of the HardyWeinberg equilibrium and the linkage equilibrium. Comparing the two two-stage approaches with the one-stage approach, that is the QPDT, by carefully choosing $L$, both of the two-stage approaches are more powerful than the one-stage approach in all the cases. However, the power of the two-stage approaches depends on $L$, the number of markers retained in the first stage, and the optimal value of $L$ depends on the LD structure of the genotypes. Steen et al ${ }^{1}$ suggested using $L=10$. Our results for the genotypes generated under linkage equilibrium suggest a similar value of $L$. A value between 10 and 20 is a good choice for $L$. However, in the more realistic situation where there is LD between markers, our results suggest a much larger $L$. It seems that the best choice for $L$ is between 100 and 500 . The reason may be that, in this case, several markers may have a strong LD with the disease locus and thus have small p-values. If we only retain a few markers in the first stage, we may pick up one or more markers that have strong LD with the disease locus but not the disease locus itself.

\section{Discussion}

The main purpose of this article is to extend the two-stage method proposed by Steen et al $^{1}$ so that the method can
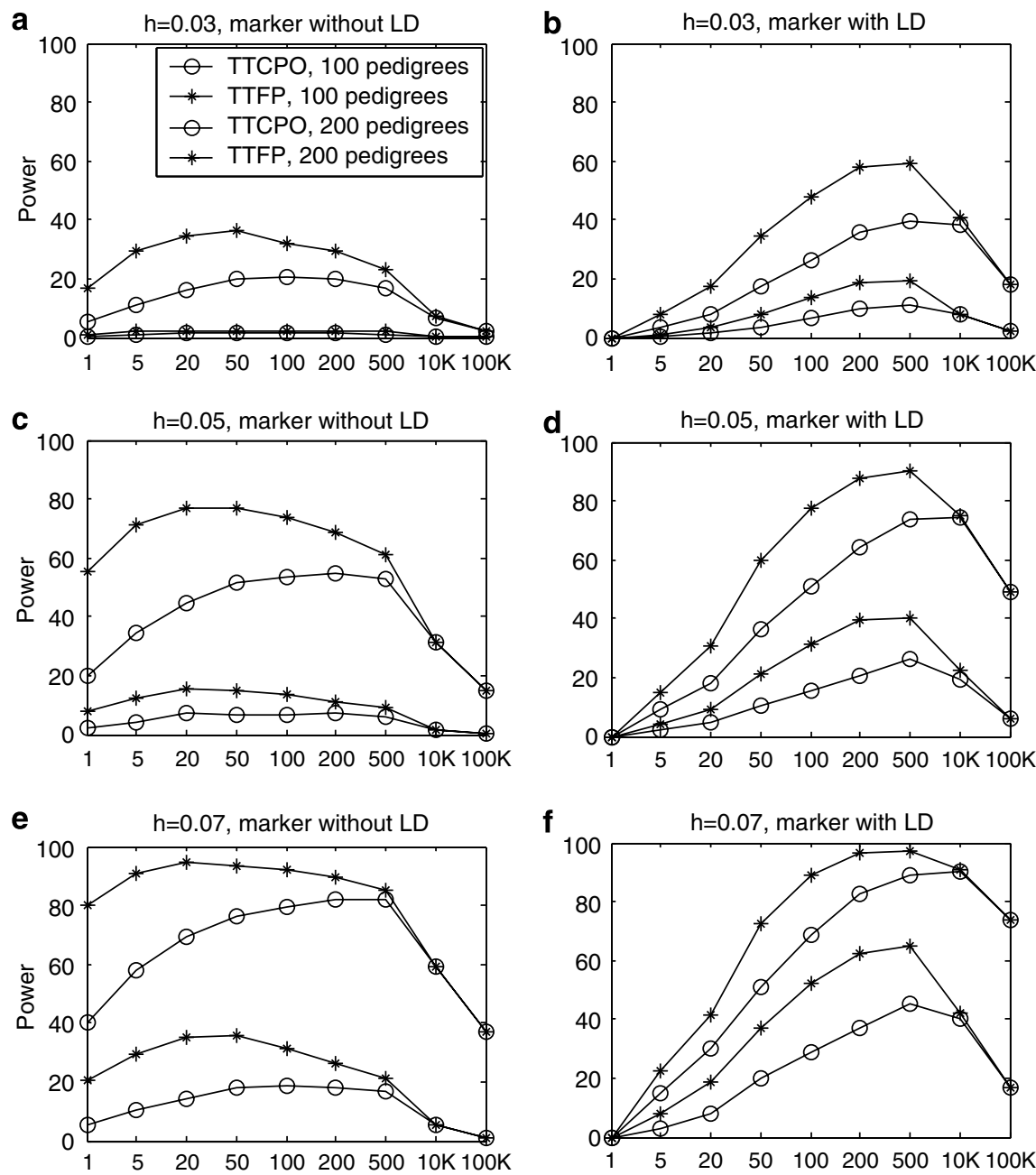

Figure 3 Power comparisons of the two-stage tests using 100 and 200 pedigrees. The $x$-axis denotes the number of markers retained by the screening test in the first stage. The total number of markers is 100000 (100K). Figures in the left panel, a, c and e, show powers based on genotype data generated under the assumption of linkage equilibrium between markers. Figures in the right panel, $\mathbf{b}$, $\mathbf{d}$ and $\mathbf{f}$, show powers based on genotype data generated by using ms software. $h$ denotes the heritability. 
incorporate parental information and can be easily applied to large pedigree data. For this purpose, we proposed two two-stage approaches, the TTCPO and TTFP. The basic assumption to guarantee the correct type I error rates of a two-stage approach is that the test used in the first stage is statistically independent of the test used in the second stage. The TTCPO, which uses children's phenotypes only, and the TTFP, which uses phenotypes of children as well as the founders in the pedigrees, have correct type I error rates in all the cases we considered. We further compare the power of the two valid approaches, the PPCPO and TTFP. Our results show that by incorporating the founders' phenotypes, the TTFP is consistently more powerful than the PPCPO. In some cases, the power of the TTFP is several times more than that of the PPCPO.

Although most of genome-wide association studies use a case-control design, genome-wide family-based association studies are still necessary. ${ }^{24}$ Family-based genomewide association studies usually use nuclear families extracted from pedigrees of previous linkage studies. The phenotype data of the nuclear families from previous linkage studies often include parental phenotypes. In this case, our proposed TTFP is feasible and more powerful than the method proposed by Steen et al. ${ }^{1}$ Although general pedigrees have not been seen in genome-wide association studies, they may be used in the follow-up studies, and thus our method is necessary.

One remaining question is choosing the value of $L$, the maximum number of markers retained in the first stage. Our simulation results show that there is no unique answer in choosing an optimal $L$. If there is weak LD between adjacent markers, the values between 10 and 20 may be a good choice for $L$. If there is strong LD between adjacent markers, the optimal value of $L$ should be much larger, 100-500 as suggested by our simulation study. In conclusion, we need further investigations on choosing the optimal value of $L$.

\section{Acknowledgements}

This work was supported by National Institute of Health (NIH) Grants R01 GM069940, RO3 HG 003613, R01 HG003054, and R03 AG024491.

\section{References}

1 Steen KV, McQueen MB, Herbert A et al: Genomic screening and replication using the same data set in family-based association testing. Nat Genet 2005; 37: 683-691.

2 Risch N: Searching for genetic determinants in the new millennium. Nature 2000; 405: 847-856.
3 Hirschhorn JN, Daly MJ: Genome-wide association studies for common diseases and complex traits. Nat Rev Genet 2005; 6: 95-108.

4 Benjamini Y, Hochberg Y: Controlling the false discovery rate: a practical and powerful approach to multiple testing. J R Stat Soc B 1995; 57: 289-300.

5 Satagopan JM, Elston RC: Optimal two-stage genotyping in population-based association studies. Genet Epidemiol 2003; 25: $149-157$.

6 Wang H, Thomas DC, Peer I, Stram DO: Optimal two-stage genotyping designs for genome-wide association scan. Genet Epidemiol 2006; 30: 356-368.

7 Lange C, Demeo D, Silvermen EK, Weiss ST, Laird NM: Using the noninformative families in family-based association tests: a powerful new testing strategy. Am J Hum Genet 2003; 73: 801-811.

8 Zhang S, Zhang K, Li J, Sun FZ, Zhao H: Test of linkage and association for quantitative traits in general pedigree: the quantitative pedigree disequilibrium test. Genet Epidemiol 2001; 18 (Suppl 1): 370-375.

9 Spielman RS, Ewens WJ: A sibship test for linkage in the presence of association: the sib transmission/disequilibrium test. Am J Hum Genet 1998; 62: 450-458.

10 Bickeboller H, Clerget-Darpoux F: Statistical properties of the allelic and genotypic transmission/disequilibrium test for multiallelic markers. Genet Epidemiol 1995; 12: 865-870.

11 Sham PC, Curtis D: An extended transmission/disequilibrium test (TDT) for multi-allele marker loci. Ann Hum Genet 1995; 59: 323-336.

12 Schaid DJ: General score tests for associations for genetic markers with disease using cases and their parents. Genet Epidemiol 1996; 13: $423-449$.

13 Sun F, Flanders WD, Yang Q, Khoury MJ: Transmission disequilibrium test (TDT) when only one parent is available: the 1-TDT. Am J Epidemiol 1999; 150: 97-104.

14 Martin ER, Monks SA, Warren LL, Kaplan NL: A test for linkage and association in general pedigree: the pedigree disequilibrium test. Am J Hum Genet 2000; 67: 146-154.

15 Allison DB: Transmission-disequilibrium test for quantitative traits. Am J Hum Genet 1997; 60: 676-690.

16 Rabinowitz D: A transmission disequilibrium test for quantitative trait loci. Hum Hered 1997; 47: 342-350.

17 Schaid DJ, Rowland CR: The use of parents, sibs, and unrelated controls to detection of associations between genetic markers and disease. Am J Hum Genet 1998; 63: 1492-1506.

18 Monks SA, Kaplan NL: Removing the sample restrictions from family-based test of association for quantitative trait locus. $A m J$ Hum Genet 2000; 66: 576-592.

19 Zhang S, Sha Q, Chen HS, Dong J, Jiang R: Transmission/ disequilibrium test based on haplotype sharing for tightly linked markers. Am J Hum Genet 2003; 73: 566-579.

20 Morley M, Molony CM, Weber T et al: Genetic analysis of genome-wide variation in human gene expression. Nature 2004; 430: $743-747$.

21 Hudson RR: Generating samples under a Wright-Fisher neutral model of genetic variation. Bioinformation 2002; 18: 337-338.

22 Nordborg M, Tavare S: Linkage disequilibrium: what history has to tell us? Trends Genet 2002; 18: 83-90.

23 Kimmel G, Shamir R: A fast method for computing high significance disease association in large population-based studies. Am J Hum Genet 2006; 79: 481-492.

24 Clerget-Darpoux F, Elston RC: Are linkage analysis and the collection of family data dead? Prospects for family studies in the age of genome-wide association. Hum Hered 2007; 64: 91-96. 